\title{
Relação de pareceristas ad hoc em 2018
}

\section{Participaram da avaliação dos artigos publicados nas edições do volume 27 da Revista de Educação Pública}

Adelina de Oliveira Novaes (Centro Internacional de Estudos em Representaçóes Sociais e Subjetividade - Educação (CIERS-ed), do Departamento de Pesquisas Educacionais, da Fundação Carlos Chagas, São Paulo/SP, Brasil)

Adir Casaro Nascimento (UCDB, Campo Grande/MS, Brasil)

Ana Rosa Costa Picanço Moreira (UNESA/UFJF, Juiz de Fora/MG, Brasil)

André Luiz Paulilo (Unicamp/Campinas/SP, Brasil)

Andreia Dalcin (UFRGS/Porto Alegre/RS, Brasil)

Ariane Franco Lopes da Silva (UFFS/SC, Brasil)

Alexandra Lima da Silva (UERJ, Rio de Janeiro/RJ, Brasil)

Alexandre de Gusmão Pedrini (UERJ, Rio de janeiro/RJ, Brasil)

Alzira Batalha Alcântara (UERJ, Rio de Janeiro/RJ, Brasil)

Amadeu José MontagniniLogarezzi (UFSCar, São Carlos/SP, Brasil)

Amailson Sandro Barros (UFMT, Cuiabá/MT, Brasil)

Betânia Laterza Ribeiro (UFU, Uberlândia/MG, Brasil)

Bruno Adriano Rodrigues Silva (UFL, Lavras/MG, Brasil)

Cândida Soares da Costa (UFMT, Cuiabá/MT, Brasil)

Carlos Edinei Oliveira (Unemat, Barra do Bugres/MT, Brasil)

Carlos Rinaldi (UFMT, Cuiabá/MT, Brasil)

Célia Maria Borges (UFU, Uberlândia/MG, Brasil)

Cláudio José Sooma Silva (UFRJ, Rio de Janeiro/RJ, Brasil)

Cristiano Maciel (UFMT, Cuiabá/MT, Brasil)

Daniel Fonseca de Andrade (UERJ, Rio de Janeiro/RJ, Brasil)

Daniela Franco Carvalho (UFU, Uberlândia/MG, Brasil)

Delarim Martins Gomes (UFMT, Cuiabá/MT, Brasil)

Edson Caetano (UFMT, Cuiabá/MT, Brasil)

Elaine Rodrigues (UEM, Maringá/PR, Brasil)

Elielson Ribeiro de Sales (UFPA, Belém/PA, Brasil)

Eliane Rose Maio (UEM, Maringá/PR, Brasil)

Elisabet Aguirre (Centro Universitário de Várzea Grande/MT, Brasil)

Emerson da Silva Ribeiro (UNIR, Ji-Paraná/RO, Brasil)

Erica Nayla Harrich Teibel (Secretaria Municipal de Saúde/Cuiabá/MT, Brasil)

Elisabeth Andrade (Regional Integrada do Alto Uruguai e das Missōes - URI/ Edu campus Frederico Westphalen) 
Elizabeth Madureira Siqueira (UFMT, Cuiabá/MT, Brasil)

Elizabeth Moreira dos Santos Schmidt (Unesp, Botucatu/SP, Brasil)

Eloy Alves Filho (UFV, Viçosa/MG, Brasil)

Fátima Aparecida da Silva Iocca (UNEMAT, Cáceres/MT, Brasil)

Fátima Elizabeti Marcomin (UNISUL, Tubarão/SC, Brasil)

Fernanda Wanderer (UFRGS, Porto Alegre/RS, Brasil)

Fernanda Cândido Magalhães (UFMT, Cuiabá/MT, Brasil)

Gerson de Souza Mól (UnB, Brasília/DF, Brasil)

Hilda Maria Silva (UNESP, Franca/SP, Brasil)

Helena Amaral da Fontoura (UERJ, Rio de Janeiro/RJ, Brasil)

Henrique Oliveira Lee (UFMT, Cuiabá/MT, Brasil)

Imara Pizzato Quadros (IFMT, Cuiabá/MT, Brasil)

Irineu Tamaio (UnB, Brasília/DF, Brasil)

Isabel Maria Sabino de Farias (UECE, Fortaleza/CE, Brasil)

Ivo Dickmann (UFPR/Londrina, Brasil)

IdemarVizolli (Universidade Federal do Tocantins - UFT, Palmas,TO)

Jader Janer Moreira Lopes (UFF, Niterói/RJ, Brasil)

Jacqueline Priego-Hernandez (Bournemouth University, UK)

Jane Teresinha Domingues Cotrin (UFMT, Cuiabá/MT, Brasil)

Jean Carlos Miranda da Silva (UFF, Niterói/RJ, Brasil)

Jorgelina Di Iori (Universidade de Buenos Aires, Buenos Aires, Argentina)

José Carlos Araújo (UFU/Uberlândia, Brasil)

José Licínio Backes (UCDB/Campo Grande, MS)

Joaquim António Sousa Pintassilgo (Universidade de Lisboa, Lisboa, Portugal

Kamila Lockmann (UFRS/Porto Alegre/RS, Brasil)

Kátia Morosov Alonso (UFMT, Cuiabá/MT, Brasil)

Kécio Gonçalves Leite (UNIR, Ji-Paraná/RO, Brasil)

Lia Ciomar Macedo de Faria (UERJ, Rio de Janeiro/RJ, Brasil)

Luci T. M. dos Santos Bernardi (UNOCHAPECÓ, Chapecó/SC, Brasil)

Lúcia Shiguemi Izawa Kawahara (SEMA, Cuiabá/MT, Brasil)

Lilian Auxiliadora Maciel Cardoso (UFMT, Cuiabá/MT, Brasil)

Luiz Augusto Passos (UFMT, Cuiabá/MT, Brasil)

Luiz Marcelo Tavares de Carvalho (UFL, Lavras/MG, Brasil)

Luiz Alexandre Barbosa Freitas (UFMT, Cuiabá/MT, Brasil)

Marlene Gonçalves (UFMT, Cuiabá/MT, Brasil)

Marco Antonio Leandro Barzano (UEFS, Feira de Santana/BA, Brasil)

Marcos Francisco Borges (UEMT, Cáceres/MT, Brasil)

Marisa Rosani Abreu da Silveira (UFPA, Belém/PA, Brasil) 
Marcos Pinheiro Barreto (UFF, Niterói/RJ, Brasil)

Maria Aparecida Rezende (UFMT, Cuiabá/MT, Brasil)

Maria Celia Borges (UFU, Uberlândia/MG, Brasil)

Maria das Graças Martins da Silva (UFMT, Cuiabá/MT, Brasil)

Marijâne Silveira da Silva (UNIR, Porto Velho/RO, Brasil)

Maristela Angotti (UNESP, Araraquara/SP, Brasil)

Naiara dos Santos Nienow (UNIR, Ji-Paraná/RO, Brasil)

Neil Franco Pereira de Almeida (UFJF, Juiz de Fora/MG, Brasil)

Nelma Baldin (Univille, Joinville/SC, Brasil)

Nileide Souza Dourado (UFMT, Cuiabá/MT, Brasil)

Osvaldo Mariotto Cerezer (Unemat, Cáceres/MT, Brasil)

Patricia dos Santos Begnami (Fundação Hermínio Ometto, Araras-SP, Brasil)

Pedro Franco de Sá (Universidade do Estado do Pará, Belém/PA, Brasil)

Regina Aparecida Silva (UFMT, Cuiabá/MT, Brasil)

Rachel Duarte Abdala (UNITAU, Taubaté/SP, Brasil)

Ricardo Castaño Gavíria (FAEBA, Salvador/BA, Brasil)

Risomar Alves dos Santos (UFCG, Campina Grande/PB, Brasil)

Rodolfo Antônio de Figueiredo (UFSCar, São Carlos, Brasil)

Rodrigo Manoel Dias Da Silva (UVRS, São Leopoldo/RS, Brasil)

Romilda Teodora Ens (PUC-PR, Curitiba/PR, Brasil)

Ronaldo Eustáquio FeitozaSenra (IFMT, Jaciara/MT, Brasil

Rosângela Kátia Sanches Mazzorana Ribeiro(UFMT, Cuiabá/MT, Brasil)

Rosemeire de Araujo Rangni (UFSCar/São Carlos/SP, Brasil)

Rute Cristina Domingos da Palma (UFMT, Cuiabá/MT, Brasil)

Sandra Pavoeiro Tavares Carvalho (UFMT, Cuiabá/MT, Brasil)

Sérgio Inácio Nunes (UFU, Uberlândia/MG, Brasil)

Simone Silveira Amorim (Unit, Brasil)

Sílva Maria dos Santos Stering (Univag/Várzea Grande/MT, Brasil)

Silas Borges Monteiro (UFMT, Cuiabá/MT, Brasil)

Soler Gonzalez (UFES, Vitória/ES, Brasil)

Suely Dulce Castilho (UFMT, Cuiabá/MT, Brasil)

Sueli Teresinha de Abreu Bernardes (UB, Uberaba/MG, Brasil)

Stélio João Rodrigues (SENAI, Regional/Blumenau/SC, Brasil

Taciana Mirna Sambrano (UFMT, Cuiabá/MT, Brasil)

Vanessa Dias Moretti (UNIFESP, São Paulo/SP, Brasil)

Vera Lúcia Gaspar da Silva (UESC, Santa Catarina/SC, Brasil)

Victor de Araújo Novicki (UCP/Petrópolis/RJ, Brasil) 